\title{
The Research of the Quality Supervision and Management Data Platform Design in the Whole Production Process for Rail used in Railway Transportation
}

\author{
Xiaodong Hao, Yong Ma, Zihao Yin* and Feng Chen \\ China Iron and Steel Research Institute Group, Beijing, China \\ *yinzihao163@163.com
}

\begin{abstract}
This paper elaborates on the research results of system architecture design and typical module design of the quality supervision and management data platform for rail used in railway transportation in the whole production process, and clarifies the function and application of the platform. The system traces the whole process of production, quality inspection, labeling and palletizing of the rails, ensuring the accuracy of rail quality traceability. Based on information traceability through the full product life cycle including sales and delivery, production process information, and material supply, the cause of quality problems can be quickly identified.
\end{abstract}

Keywords: quality supervision; data platform; transportation; rails; system architecture design; typical module design.

\section{Preface}

In the whole production process, quality supervision and management of rail used in railway transportation start from the refined molten steel in steel making, to the follow-up quality tracking of continuous casting billet, billet heating, billet rolling, pre-bending, straightening, nondestructive testing, on-line inspection, finishing, rail end heat treatment, full-length heat treatment, rail finished product labeling to finished product stacking, and the whole system supervision and feedback. The rail quality supervision and management data platform is the foundation of rail production, use and recovery. Based on the construction of rail quality supervision and management data platform in the whole process, that can be realized: 1) serving the quality supervision for railway personnel stationed in-plant, strengthen the quality supervision and management functions, and comprehensively guaranteeing the welding quality of rails, turnout and rails; 2) managing and utilizing quality data to support the deepening of quality supervision services and provide decision support for quality cause analysis and retrospective search; 3) collecting and analyzing the report of quality guarantee and welding rail production data of steel mills intensively, and getting through the rail life cycle management process; 4) reducing labor intensity and improving detection accuracy. Therefore, the rail quality supervision and management data platform is an important research direction in the field of railway transportation at home and abroad.

\section{System Architecture Design}

The railway rail quality whole-process supervision and management data platform covers the rail manufacturing process and rail operation and maintenance process, with the four modules of quality management, data modeling, process management and system integration as the main functional blocks, as show in Fig. 1, combining the remote app method with the whole-life cycle control, integrating the MES system of the upstream iron and steel enterprise and the downstream in-plant inspection system to form a whole-process management platform for data interaction, mining and analysis.

According to the characteristics of the whole process and multi-system and the technological requirements of each process, the platform integrates construction management and security measures, including remote login identity confirmation, operation records, authority setting, terminal backup, etc. as the main content and supplement of system security measures, thus improving the overall security level of the system [1]. 


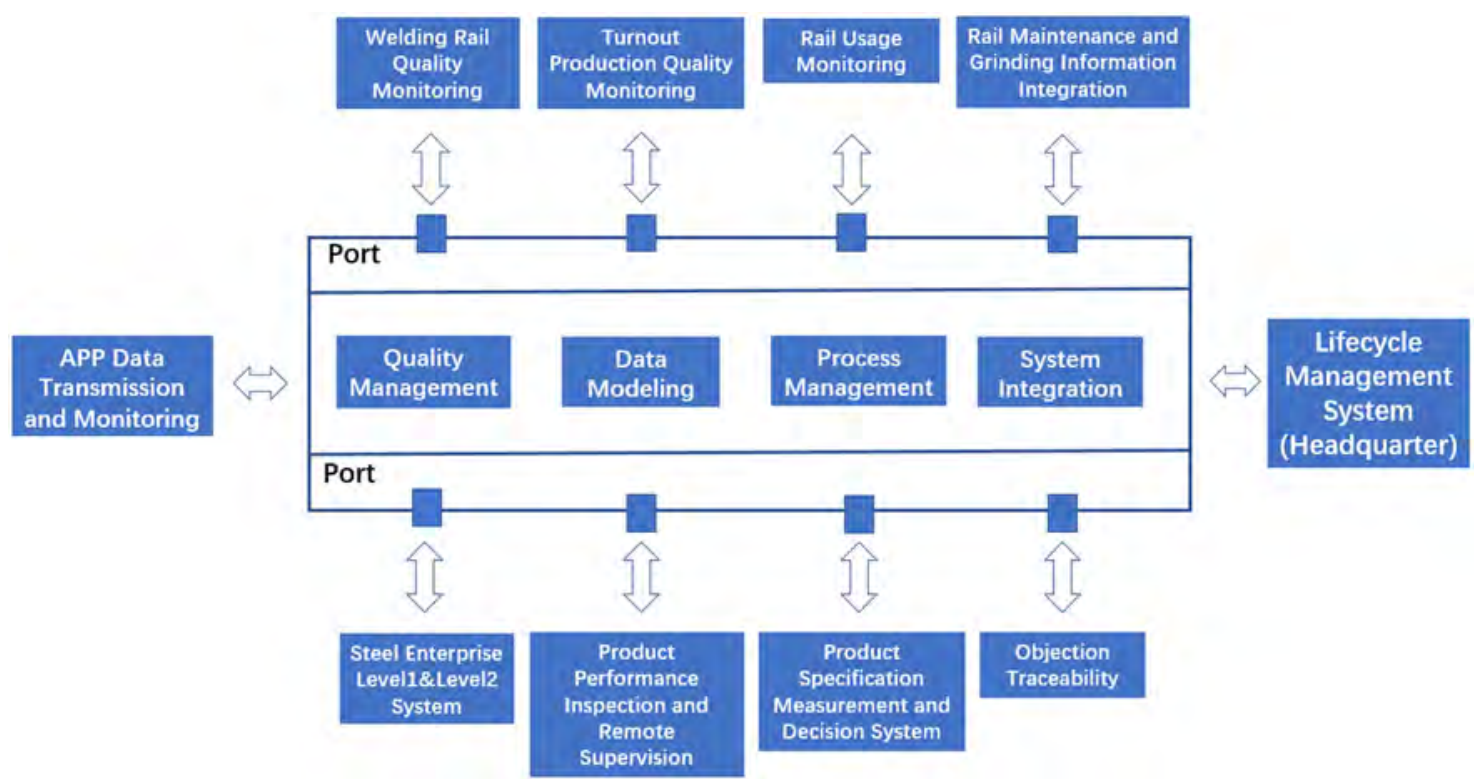

Figure 1. Basic Structure of Data Platform for The Whole Process Quality Supervision and Management of Rail Used in Railway Transportation

\section{Research on Typical Module Design}

\subsection{Production Planning Management and Docking of Iron and Steel Enterprises}

The confirmation of steel production plan imported into the platform is a management step that must be taken before production can be released. The plan confirmation function mainly includes three aspects: 1) the executives will confirm the planned process route and determine the planned date for each operation. 2) the operator can check the feasibility of the instruction plan date and adjust the production path if necessary according to the unit operation capacity of each process, the unit maintenance plan and inventory. 3) when the process capacity and inventory logistics can be balanced, the plan should be confirmed and the production can be officially released. Plan tracking is the core function of instruction management and an important basis for the platform to accept product information from steel enterprises. Its essence is to track the whole process of instruction execution in real time. The plan tracking fully accepts the event data from other related subsystems at various function points, and processes the event data according to strict calculation logic, finally reflecting the dynamic changes of the command process shortage, material inventory and process throughput, among which the instruction process shortage is the key data necessary to organize production according to the instructions. According to the shortage of instruction process, the instruction status can be determined and the instruction process can be mastered.

\subsection{Performance Collection and Management of Laboratory and Field Products in Iron and Steel Enterprises}

The platform collects and manages the inspection results uploaded by L2 to make quality final judgment basis for the system. Data collection and management of on-site inspection items, including flatness detector and surface inspection, upload and manage instrument inspection values in real time. The relevant inspection and test result data obtained from each process L2 can be queried as inspection and test performance data.

The quality inspection personnel will carry out final judgment on the billet and rod and wire that have passed the surface quality judgment. The billet will be judged on the basis of finished samples and refined samples, and the rod and wire will be judged on the basis of initial samples and duplicate samples. The final judgment can be judged as qualified or unqualified and uploaded to the platform. The platform can also be extended to judge the steel grade according to the finished product sample, composition standard and abnormal furnace times. For the undetermined steel grade, the second final 
judgment can be made after the previous judgment, and will make feedback and backup. The process can reduce manual intervention, reduce labor intensity and improve the data accuracy.

\subsection{Product Quality Traceability}

Based on multi-dimensional statistical process control, the platform adopts a product trackingmodel reflecting the evolution process of material flow in the whole process to realize material flow correlation, hierarchical query and backtracking matching among different processes, and adopts data collection technology in different time and space dimensions such as product, time and event to achieve accurate time and space matching of information flow and material flow data in the whole process. The module functions contain fuzzy tracing and precise tracing. Fuzzy tracing refers that we only need get information about one or more batches of raw materials corresponding to one batch of products. Accurate traceability refers to the raw material batch that can be traced back to the single finished product, and when the raw material batch is switched, it can also be traced back to the product that was produced when the raw material batch was switched.

Accurate traceability can help steel enterprises, operation and maintenance enterprises, quality management personnel and process developers to minimize the problem products to the largest extent and control the quantity of the problem products after finding quality problems. It supports the tracing of information from sales and delivery, production process information, to material supply throughout the product life cycle, so as to quickly locate the causes of quality problems. Input query interface is also provided. If the user inputs any product number, serial number, batch number, production date, receipt date and shipment date, the detailed data of the product in each process can be obtained, and the corresponding results can be queried by setting different conditions so as to realize the forward and backward traceability of the product.

\subsection{Interface Design}

Data exchange between platform application system communication and communication with other application systems is necessary and critical. The quality of data exchange design will affect the implementation progress, degree of implementation and final operation effect of the system. The communication between rail quality supervision and management platform and other application systems will adopt XML mode and database-based communication mode as appropriate. Since communication between application systems may be affected by network failures, host failures, application system failures, etc., one problem that cannot be ignored in communication is data protection when communication is abnormal.

Interface management functions include: interface management with level 2 computer systems in each process, receiving real-time data, process data, consumption data and test data collected by level 2 systems; downward transmission of L3 system plan and quality information to level 2 computer system; Interface management with MES system of each process; Interface with related robot system and measuring equipment; reserve interface management with the host system.

The data communication of the platform will adopt caching mechanism to solve the problem of communication interruption caused by external system failure within a limited time. All exchange data sent to other application systems will be stored in the database and a field will be designed to indicate the sending status, with the initial value of " unsuccessful". When the data receiver feedbacks successful reception, the sending status is set to " successful". When the communication system recovers from the failure, it will automatically check the cached data with the sending status of " unsuccessful" and automatically resend the data. Production quality management will design and reserve data exchange interface with host systems.

\section{Conclusion}

The " Three - Year Action Plan to Enhance the Core Competitiveness of Manufacturing Industry (2018-2020)" (Department of Industry of NDRC [2017] No. 2000) announced by China clearly proposes to " build a full life cycle management system to meet the needs of intelligent data 
processing, analysis and maintenance decision-making and improve the level of intelligent operation and maintenance support for high-speed railway infrastructure [2] ". The data platform of rail quality supervision and management in the whole process is the foundation of rail life cycle management system, connecting the whole process management links of steel, transportation, operation and maintenance, effectively reducing the labor intensity and improving the management efficiency and accuracy.

\section{References}

[1]. W.F. Zhang, Architecture of intelligent integrated monitoring system for urban rail transit, China Railway Science 27 (2006), 126-132.

[2]. Implementation Plan of Key Technology Industrialization of Rail Transit Equipment. Development and Reform Commission., 2017. 